\title{
An ounce of prevention would go a long way for depression in Canada
}

\author{
Devin Mahnke
}

\section{University of Calgary}

In the Diagnostic and Statistical Manual of Mental Disorders (DSM-IV-TR), Major Depressive Disorder (MDD) is characterized by symptoms of sadness, loss of interest, fatigue, sleep or appetite disturbance, guilt, and suicidal ideation. ${ }^{1}$ A diagnosis of MDD is given if at least five symptoms, including sadness or loss of interest, persist for at least two weeks. Under these criteria, depression afflicts one in 10 Canadians at some point in their lives. While prevalence estimates are more variable in young people due to heterogeneity in age range and diagnostic paradigm (symptoms can present differently and be more difficult to identify in young people), a recent study indicates that depression afflicts about $8 \%$ of adolescents. ${ }^{2}$ Despite the considerable burden of depression on youth, evidence indicates that they are presently underrepresented in mental health research and services. ${ }^{3}$ Although numerous advances have been made in pharmacology, psychotherapy, and other therapeutic approaches, depression remains a large and undertreated burden on Canada's health care system. The prevailing discussion of depression, which includes information provided by organizations like Health Canada, focuses on risk factors outside of the individual's control, such as genetic predisposition, financial hardship, and death of a loved one. ${ }^{4}$ It is important to acknowledge that public health may be better served by focusing on the modifiable social and lifestyle factors that contribute to MDD, in addition to acknowledging the impact of extrinsic factors. ${ }^{5}$ Identifying and treating depressive symptoms as early as possible with a focus on preventative approaches could greatly benefit Canada's economy and the health of our population.

The small things add up, and youth in particular could benefit from increased awareness of, and emphasis on, depression-related factors within their control. This point is especially relevant considering the reluctance of young people, and their parents and healthcare providers, to use antidepressant medications as a first-line treatment for depression. ${ }^{6} \mathrm{~A}$ host of lifestyle factors contribute to increasing risk of depressive illness, including stress, obesity, sleep deficit, lack of exercise, and social isolation. ${ }^{5}$ Furthermore, clinical data suggest that depression is better characterized as a continuous spectrum, ${ }^{1}$ as opposed to the clean break between depression and wellness implied by the DSM-IV. The evidence supports adopting a societal view of depression as a state strongly influenced by personal and social health, which can affect nearly anyone given the right circumstances, rather than as a disease with which certain people are afflicted. A focus on modifiable factors could provide symptom abatement and quality-of-life improvement for some sufferers of depression, ${ }^{5}$ improving public health overall and leaving the healthcare system to intervene only when it is truly needed.

The lion's share of the study and treatment of depression goes to adult populations, but the disorder is relatively more prevalent in youth, ${ }^{3}$ being one of the chief causes of disease burden in individuals aged $15-24 .{ }^{6}$ Successful treatment of adolescent depression not only serves to alleviate suffering and risk of suicide in young people directly, but could also curb the incidence of continued mental illness, alcoholism, and drug abuse stemming from unresolved depression. ${ }^{6}$ Considering childhood or adolescent depression is a strong determinant for continued mental illness and substance abuse incidence, it is important to note that early intervention in young people may potentially be more impactful than similar intervention in adults. Furthermore, recent epidemiological research supports a 'staging model' of mental illness, by which more serious disorders can potentially be predicted by subclinical symptoms of depression during adolescence. ${ }^{7}$ From the perspective of public health promotion, addressing the mental health needs of young people stands to benefit everyone. From an economic perspective, although the cost of depression 
is largely manifested as lost productivity in the workforce, ${ }^{8}$ the most cost-effective solution will be prevention of the disorder before entry into the workforce. ${ }^{9}$

In combating depression, research indicates the importance of early intervention and preventative strategies, which will require increased awareness of the importance of youth mental health as well as expansion of youth mental health services. Renowned psychiatrists Dr. Stanley Kutcher and Dr. Patrick McGorry, major proponents of early intervention strategies, argue that what is needed is a concerted effort in addressing youth mental health through specific policy changes $^{8}$ and introduction of youth-friendly treatment programs, which may be most effective if integrated into the school system. ${ }^{9,10}$ These are sweeping changes that will require a large investment of time and effort to implement, ${ }^{8}$ but the evidence suggests that the cost of continuing to inadequately address the mental health needs of youth would be far greater. The guidelines to improving early detection and treatment have been laid out. ${ }^{7-10}$ What remains is to appreciate the importance of preventing depression in young people, and act on it.

\section{References}

1. Kendler KS, Gardner CO, Jr. Boundaries of major depression: an evaluation of DSM-IV criteria. Am J Psychiatry. 1998 Feb;155(2):172-7.

2. Cheung, AH, Dewa, CS. Canadian Community Health Survey: Major Depressive Disorder and Suicidality in Adolescents. Can J Psychiatry. 2007 Apr;52(4):228-32.

3. Patton GC, Hetrick SE, McGorry P. Service responses for youth onset mental disorders. Curr Opin Psychiatry. 2007 Jul;20(4):319-24.

4. It's Your Health: Health Canada 2009. Report No.: 978-1-100-10340-2.

5. Jacka FN, Mykletun A, Berk M. Moving towards a population health approach to the primary prevention of common mental disorders. BMC Med. 2012;10:149.

6. Patel V, Flisher AJ, Hetrick S, McGorry P. Mental health of young people: a global public-health challenge. Lancet. 2007 Apr 14;369(9569):1302-13.

7. Hickie IB, Scott EM, Hermens DF, Naismith SL, Guastella AJ, Kaur M, et al. Applying clinical staging to young people who present for mental health care. Early Interv Psychiatry. 2013 Feb;7(1):31-43.

8. Kutcher S, Hampton MJ, Wilson J. Child and adolescent mental health policy and plans in Canada: an analytical review. Can J Psychiatry. 2010 Feb;55(2):100-7.

9. McGorry PD. Is early intervention in the major psychiatric disorders justified? Yes. BMJ. 2008;337:a695.

10. Kutcher S, Wei Y. Mental health and the school environment: secondary schools, promotion and pathways to care. Curr Opin Psychiatry. 2012 Jul;25(4):311-6.

\section{Devin Mahnke}

Devin is a Master's student in Neuroscience at the University of Calgary. He is interested in understanding how mental illness affects the structure and function of the brain, and is currently using magnetic resonance imaging to study Bipolar Disorder and Major Depressive Disorder. 